\title{
Efecto del Heliocarpus americanus (palo de goma) como adhesivo para prótesis dental removible
}

\author{
Effect of Heliocarpus Americanus (Palo de Goma) as an Adhesive for Removable \\ Dental Prostheses
}

\author{
Nancy Doris Calzada Gonzales « 1,a , Maribel Jessica Calzada Gonzales 2,b , Marisol Rossana Ortega Buitrón 2,a , \\ Juana Irma Palacios Zevallos $2, c$.
}

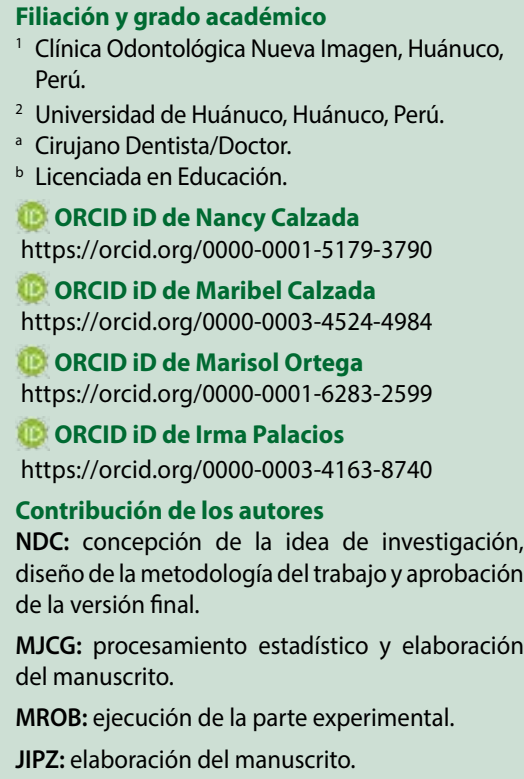

\section{RESUMEN}

Objetivo. Evaluar el efecto del Heliocarpus americanus (palo de goma) como adhesivo para prótesis. Métodos. Estudio experimental, prospectivo, transversal. El tamaño de la muestra se calculó por fórmula de comparación de proporciones para grupos independientes, siendo incluidos 32 sujetos en dos grupos de: 16 pacientes para el grupo de experimental (GE) "palo de goma" (Heliocarpus americanus), y 16 pacientes para el de control (GC) "crema adhesiva Corega". Para evaluar el efecto del adhesivo, primero se llevó a cabo la clasificación taxonómica de la corteza, análisis fitoquímico, y espectrofotometría infrarroja, realizado en la Universidad Nacional Mayor de San Marcos, así como la elaboración del gel adhesivo. Ocho horas después de la aplicación del gel adhesivo, se midió la estabilidad y retención utilizando el método de Woelfel, así como el grado de aceptabilidad de acuerdo a la escala Hedónica. Por último, se aplicó la prueba suma de rangos de Wilcoxon. Resultados. El análisis estadístico no mostró diferencia significativa ( $p>0,05$ ) entre el (gel de Heliocarpus americanus y la crema adhesiva Corega ${ }^{\circledR}$ GlaxoSmith. El grado de aceptabilidad en el grupo experimental muestra un alto porcentaje en "ni me gusta ni me disgusta" (37,5 \%). Conclusión. El gel experimental no presenta diferencias significativas en la estabilidad y retención de la prótesis total. Por lo tanto, el gel adhesivo se puede usar de manera efectiva como adhesivo para prótesis total.

Palabras clave: humanos, tamaño de la muestra, estadísticas no paramétricas, grupos de control, adhesivos, corteza vegetal, estudios prospectivos, estudios transversales, extremidades artificiales, diseño de la investigación, implantación de prótesis, espectrofotometría, infrarrojos, fitoquímicos (Fuente: DeCS - BIREME).

\section{ABSTRACT}

Objective. To evaluate the effect of Heliocarpus americanus (Palo de Goma) as an adhesive for prosthetics. Methods. Experimental, prospective, crosssectional study. Sample size was calculated by the proportions comparison formula for independent groups, 32 subjects being included in two groups of: 16 patients for the experimental group (EG) "palo de goma" (Heliocarpus americanus), and 16 patients for the control group (CG) "Corega adhesive cream". To evaluate the effect of the adhesive, the taxonomic classification of the bark, phytochemical analysis, and infrared spectrophotometry were first carried out at Universidad Nacional Mayor de San Marcos, as well as the elaboration of the adhesive gel. Eight hours after the application of the adhesive gel, the stability and retention were measured using the Woelfel method, as well as the degree of acceptability according to the Hedonic scale. Finally, the Wilcoxon rank sum test was applied. Results. The statistical analysis did not show a significant difference ( $p>0.05$ ) between the Heliocarpus americanus gel and the Corega ${ }^{\circledR}$ GlaxoSmith adhesive cream. The degree of acceptability in the experimental group shows a high percentage in "neither like nor dislike" (37.5 $\%)$. Conclusion. The experimental gel does not show significant differences in the stability and retention of total prosthesis. Therefore, the adhesive gel can be used effectively as an adhesive of the total prosthesis.

Keywords: Humans, Sample Size, Statistics Nonparametric, Control Groups, Adhesives, Plant Bark, Prospective Studies, Cross-Sectional Studies, Artificial Limbs, Research Design, Prosthesis Implantation, Spectrophotometry, Infrared, Phytochemicals (Source: MeSH - NLM). 


\section{INTRODUCCIÓN}

El problema que enfrentan los pacientes portadores de prótesis totales en la retención, constituye el riesgo de desalojo de las mismas. Los adhesivos para prótesis proporcionan una excelente retención protésica, integrándolos a la vida social con una mayor libertad de movimientos en el habla o la masticación; aumentando, en definitiva, su seguridad y autoestima. Se calcula que el $40 \%$ de los adultos mayores son desdentados; cifra que afortunadamente cada día es menor gracias a la prevención ${ }^{(1)}$.

Algunos estudios demuestran que, de la población edéntula portadora de prótesis total, aproximadamente entre un 15 y un $33 \%$ utilizan sistemas adhesivos complementarios ${ }^{(2)}$. Para el año 2017, los adultos mayores representan el 10,1\% de la población del Perú; sin embargo, datos estimados muestran que para el año 2025 estos conformarán el $13 \%$ del total de habitantes. El reporte técnico del INEI también muestra que el $77,5 \%$ de los adultos mayores han reportado tener algún problema de salud ${ }^{(3)}$.

La retención completa de la prótesis puede verse afectada negativamente por los cambios en el tejido duro y blando que comprenden la adaptación de la superficie de la dentadura al tejido oral, la disminución relacionada con la edad o la medicación en la consistencia y el volumen de la saliva, y las reducciones relacionadas con la edad en la fuerza de mordida y control neuromuscular ${ }^{(4)}$. Los adhesivos están formulados con polímeros hidrófilos sintéticos que absorben la saliva y proporcionan una capa viscosa entre la mucosa oral del tejido portador de la dentadura y la propia dentadura ${ }^{(5)}$.

Diversos autores como Psillakis et al. ${ }^{(6)}$ encontraron una mejora significativa en la fuerza de mordida hasta el desplazamiento de la dentadura postiza cuando se usó adhesivo para prótesis dentales totales y un gnatómetro. Pawan et al. ${ }^{(7)}$ demostraron el efecto de los adhesivos para prótesis dentales sobre la fuerza de mordida incisal de quienes usan dentaduras postizas completas, utilizando transductores de presión; lo que resultó ser significativamente efectivo para mejorar la fuerza de mordida incisal de las dentaduras completas hasta el desplazamiento de la dentadura superior.

De Oliveira et $a l .{ }^{(8)}$ evaluaron el desempeño masticatorio de usuarios de prótesis completas después de usar dos adhesivos y demostraron que el uso de adhesivo para prótesis dentales mejora el rendimiento masticatorio de los usuarios de dentaduras postizas completas convencionales.
Mendoza et al. ${ }^{(9)}$ analizaron el efecto de un adhesivo para dentaduras postizas sobre la satisfacción y los parámetros kinesiográficos de los usuarios completos de prótesis; lo cual mejoró la satisfacción de los pacientes y alteró los movimientos mandibulares, con aumentos en los movimientos verticales durante la masticación y menos intrusión de las dentaduras postizas completas. Figueiral et al. ${ }^{\left({ }^{10)}\right.}$ evaluaron el efecto de retención de los diferentes adhesivos para dentaduras postizas en las prótesis totales maxilares utilizando un transductor intraoral; demostraron que los adhesivos para prótesis dentales mejoran la retención de la prótesis y la estabilidad y encontrando que los pacientes perciben una mayor confianza y comodidad.

El Heliocarpus americanus es conocido en la Amazonía del Perú como palo de goma, huampo blanco, topa o balso blanco; siendo este uno de los países más megadiversos del mundo (está entre los 12 primeros) en sus diferentes nichos ecológicos, ofrece una amplia gama, tanto en flora y fauna como respecto al uso de las plantas medicinales ${ }^{(11)}$. Asimismo, es un género botánico de fanerógamas perteneciente a la familia Tiliaceae, con 43 especies originarias de Centroamérica y distribuidas desde México hasta Paraguay ${ }^{(12)}$. La mayoría de las especies son árboles y arbustos adaptables a diferentes condiciones; se encuentran tanto en zonas bajas y calientes, como en regiones templadas y frías de las montañas ${ }^{(13)}$.

Alvarado et al. demostraron que en la caracterización proximal de la corteza de huampo (Heliocarpus americanus) el dato obtenido es de un alto porcentaje de carbohidratos $(58,3 \%$ y bajo en proteínas $(0,2 \%)$, características que indica la presencia de hidrocoloide en la corteza de huampo y el uso del hidrocoloide de huampo como gelificante no tiene diferencias significativas en cuanto al sabor, comparado a la mermelada de piña convencional ${ }^{(14)}$. El objetivo principal de este estudio fue evaluar el efecto del Heliocarpus americanus (palo de goma) como adhesivo para prótesis dental removible frente a la crema adhesiva Corega.

\section{MÉTODOS}

\section{Ámbito del estudio y diseño}

El presente estudio que tuvo un diseño experimental, fue desarrollado en la clínica odontológica de la Universidad de Huánuco durante el periodo de julio a octubre del 2018. Se realizó un estudio experimental prospectivo y transversal, conformado por el Heliocarpus americanus (Grupo experimental) y la crema adhesiva Corega ${ }^{\circledR}$ GlaxoSmith (Grupo control). 
Las variables en estudio fueron: retención y estabilidad de las prótesis totales $(\mathrm{VI})$ y la crema adhesiva experimental de Heliocarpus americanus y la crema adhesiva comercial Corega (VD).

\section{Población y muestra}

La población estuvo constituida por todos los pacientes portadores de prótesis total que acudieron a la clínica odontológica de la Universidad de Huánuco, de julio a octubre del 2018. La muestra estuvo conformada por 32 sujetos en total; 16 sujetos por cada grupo. Para la determinación del tamaño muestral se utilizó el Software Epidat v.3,1 en su módulo de muestreo, cálculo de tamaño de muestra para comparación de proporciones de grupos independientes, con un nivel de confianza de $95 \%$ y un poder de prueba de $80 \%$.

Los criterios de inclusión considerados fueron pacientes de 50 a 70 años, con flujo salival disminuido, mientras que los criterios de exclusión correspondieron a pacientes portadores de prótesis total mal adaptadas.

\section{Instrumentos de medición}

El instrumento utilizado para medir la retención de las prótesis dentales fue la escala de retención, que constó de 4 preguntas: ¿la prótesis no tiene movimiento o un pequeño movimiento a la actividad masticatoria o de fonación? (alta retención), ¿la prótesis tiene un pequeño movimiento a la actividad masticatoria y un ligero movimiento en la fonación? (moderada retención), ¿la prótesis tiene un ligero movimiento a la actividad masticatoria, moderado movimiento en la fonación? (baja retención), ¿la prótesis tiene un movimiento amplio a la actividad masticatoria y fonación? (no retención). Este instrumento fue validado por 2 profesionales de la especialidad de rehabilitación y 3 expertos metodólogos; además se realizó la prueba de confiabilidad de Cronbach, cuyo valor fue 0,854 .

Para medir el grado de aceptabilidad del adhesivo se utilizó la escala hedónica que presentó 5 ítems: me gusta mucho, me gusta ligeramente, ni me gusta ni me disgusta, me disgusta ligeramente y me disgusta mucho.

\section{Proceso de recolección de datos}

Para la recolección de datos se aplicó un cuestionario relacionado con la estabilidad, retención de la prótesis total superior y el grado de aceptabilidad de las sustancias adhesivas relacionado con el maxilar superior. La estabilidad clínica de las prótesis dentales fue medida, de acuerdo al método descrito por Woelfel, con el paciente en posición fisiológica de descanso con apertura bucal. Detallado en la parte procedimental.

\section{Procedimiento clínico}

a. Treinta y dos pacientes desdentados que cumplieron con los criterios de inclusión y exclusión fueron asignados de acuerdo al muestreo aleatorio simple realizado en el programa estadístico EPIDAT versión 3.1. Los dos grupos fueron los siguientes:

- Adhesivo experimental: Heliocarpus americanus (palo de goma).

b. Adhesivo comercializado: Crema adhesiva de prótesis dental crema adhesiva Corega ${ }^{\circledR}$ GlaxoSmith.

c. La corteza de Heliocarpus americanus (palo de goma) se obtuvo del centro poblado Porvenir de Marona del distrito de Daniel Alomia Robles, provincia de Leoncio Prado, en la región Huánuco. En la facultad de Farmacia y Bioquímica de la Universidad Nacional Mayor de San Marcos se llevó a cabo la clasificación taxonómica de la corteza y el análisis de espectrofotometría infrarroja, dando como resultado la presencia de polisacárido (Celulosa modified en 81,6\%).

d. La preparación del gel: al extracto blando etanólico de Heliocarpus americanus (palo de goma), se le dio forma farmacéutica por medio de la preparación de un gel. El procedimiento consistió en medir la cantidad exacta de carbopol (1\%), propilenglicol (7\%) y metilparabeno $(0,1 \%)$ que fueron dispersados en agua destilada. Esta dispersión de carbopol se mantuvo en reposo durante 24 horas, lo que permitió su estabilización completa. A continuación, el carbopol dispersado se mezcló mediante agitación continua y en caliente hasta formar el aspecto de un gel. La dispersión obtenida se neutralizó con una cantidad adecuada de trietanolamina, hasta obtener un pH de 5,0 a 5,5. Se dejó reposar esta dispersión en un recipiente de plástico durante una semana a temperatura ambiente. Entonces, una concentración correspondiente al $10 \%$ del extracto blando Heliocarpus americanus (palo de goma) fue diluida con propilenglicol (3\%) y agua destilada en caliente y se añadió a la dispersión de carbopol. El gel obtenido fue almacenado en un recipiente de vidrio a temperatura ambiente ${ }^{(15)}$.

e. Antes de aplicar el gel experimental y las sustancias de control (crema adhesiva Corega ${ }^{\circledR}$ GlaxoSmith), se limpió con un cepillo dental Dento, con pasta dental Dento Intradevco Industrial S.A. Posteriormente, luego de secar la superficie de la prótesis, fue aplicado el gel experimental en dos pequeñas cantidades a cada lado de la cresta de la dentadura maxilar (dentro de los bordes bucolabiales y posteriores) y un toque en el medio del área palatina 
de la superficie interna de la prótesis superior y las tres pequeñas cantidades del gel adhesivo de 0,5 gramos; cada uno haciendo un total de 1,5 a $2 \mathrm{~g}$. La cantidad exacta varió individualmente según el tamaño de la prótesis dental, se indicó abstenerse del consumo de líquidos calientes durante 5 minutos, la aplicación se realizó por 8 horas y la crema adhesiva Corega ${ }^{\circledR}$ GlaxoSmith de acuerdo con las instrucciones de la etiqueta del producto.

Después de las 8 horas de aplicación del gel experimental y la crema adhesiva Corega ${ }^{\circledR}$ GlaxoSmith, los participantes completaron un cuestionario relacionado con la estabilidad, retención de la prótesis total superior y el grado de aceptabilidad de las sustancias adhesivas relacionado con el maxilar superior. La estabilidad clínica de las prótesis dentales fue medida, de acuerdo al método descrito por Woelfel, con el paciente en posición fisiológica de descanso con apertura bucal. Para realizar la aplicación, se colocaron los dedos índice y pulgar sobre la dentadura inferior aplicando una fuerza horizontal y rotatoria, determinando el grado de movimiento, que constó de 5 preguntas: ¿la prótesis no tiene movimiento o un pequeño movimiento frente a la aplicación de una gran fuerza directa o giratoria? (excelente), ¿la prótesis no tiene movimiento o es muy mínimo al aplicar una gran fuerza giratoria, pero se mueve o es desalojada cuando una gran fuerza directa es aplicada en uno de los lados o en el frente de la prótesis? (bueno), ¿la prótesis tiene un considerable movimiento cuando se aplica una fuerza giratoria y es desalojada por una fuerza directa moderada? (aceptable), ¿una fuerza suave, directa o giratoria, causa movimiento en la prótesis y es desalojada? y ¿la prótesis debe ser rebasada o cambiada? (malo).

\section{Aspectos éticos}

El estudio se realizó siguiendo los principios éticos de la investigación médica en seres humanos según la Declaración de Helsinki de la Asociación Médica
Mundial. Se obtuvo la aprobación del Comité de Ética de la Escuela Académico Profesional de Odontología de la Universidad de Huánuco.

\section{Análisis de datos}

Para realizar el análisis descriptivo e inferencial de datos se utilizó el programa estadístico IBM SPSS Statistics 23. Para comparar los dos grupos de estudios o muestras independientes se utilizó la prueba, no paramétrica, suma de rangos de Wilcoxon, debido a que cumple con los supuestos. Los datos pertenecen a dos muestras aleatorias, las observaciones son independientes y la escala de medición es, al menos, ordinal.

\section{RESULTADOS}

En la tabla 1 se presenta la distribución de las variables grupos de estudio con estabilidad de acuerdo al método Woelfel de la prótesis total. En esta muestra que el adhesivo Heliocarpus americanus prevalece el parámetro "aceptable" (50\%) en la estabilidad de la prótesis total, seguido de buena (43,2\%); mientras que en el grupo control (Corega). Los parámetros de estabilidad que presentaron mayores porcentajes fueron "aceptable" (75 \%) y "buena" $(18,3 \%)$. No se hallaron diferencias significativas en la estabilidad de las prótesis dentales ( $p=0,521)$ : adhesivo natural (Heliocarpus americanus) y adhesivo comercial crema adhesiva Corega.

En la tabla 2 se presenta la distribución de los grupos de estudio según la retención de la prótesis total. En esta se muestra que para el adhesivo Heliocarpus americanus prevalece el parámetro "modera retención" (75\%), seguido de "alta retención" (25\%); mientras que en el grupo control (crema adhesiva Corega) prevalecieron el parámetro "moderada retención" $(81,3 \%)$ seguido de "alta retención" $12,6 \%$. Siendo $p>0,05$, no se rechaza hipótesis nula; por lo tanto, la retención de la prótesis total (maxilar superior) no difiere según las sustancias experimentadas, dado que no hubo diferencias significativas ( $p=0,521)$.

Tabla 1. Estabilidad de la prótesis total de las diferentes sustancias adhesivas

\begin{tabular}{|c|c|c|c|c|c|c|}
\hline \multirow{2}{*}{$\begin{array}{l}\text { Sustancias } \\
\text { evaluadas }\end{array}$} & \multicolumn{4}{|c|}{ Estabilidad } & \multirow{2}{*}{ Total } & \multirow{2}{*}{$p$} \\
\hline & Mala & Aceptable & Buena & Excelente & & \\
\hline \multirow{2}{*}{ Heliocarpus americanus } & 1 & 8 & 7 & 0 & 16 & 0,521 \\
\hline & $6,3 \%$ & $50 \%$ & $43,2 \%$ & $0 \%$ & $100 \%$ & \\
\hline \multirow{2}{*}{ Grupo control } & 0 & 12 & 3 & 1 & 16 & \\
\hline & $0 \%$ & $75 \%$ & $18,2 \%$ & $6,3 \%$ & $100 \%$ & \\
\hline \multirow{2}{*}{ Total } & 1 & 20 & 10 & 1 & 32 & \\
\hline & $3,1 \%$ & $62,6 \%$ & $31,2 \%$ & $3,1 \%$ & $100 \%$ & \\
\hline
\end{tabular}

Suma de rangos de Wilcoxon $(p>0,05)$ no significativo. 
Tabla 2. Retención de la prótesis total de las diferentes sustancias adhesivas

\begin{tabular}{|c|c|c|c|c|c|c|}
\hline & \multicolumn{4}{|c|}{ Retención } & \multirow[b]{2}{*}{ Total } & \multirow[b]{2}{*}{$p$} \\
\hline & $\begin{array}{c}\text { No } \\
\text { retención }\end{array}$ & Mínima retención & $\begin{array}{l}\text { Moderada } \\
\text { retención }\end{array}$ & $\begin{array}{c}\text { Alta } \\
\text { retención }\end{array}$ & & \\
\hline \multirow{2}{*}{ Heliocarpus americanus } & 0 & 0 & 12 & 4 & 16 & 0,249 \\
\hline & $0 \%$ & $0 \%$ & $75 \%$ & $25 \%$ & $100 \%$ & \\
\hline \multirow{2}{*}{ Grupo control } & 0 & 1 & 13 & 2 & 16 & \\
\hline & $0 \%$ & $6,3 \%$ & $81,3 \%$ & $12,5 \%$ & $100 \%$ & \\
\hline \multirow{2}{*}{ Total } & 0 & 1 & 25 & 6 & 32 & \\
\hline & $0 \%$ & $3,1 \%$ & $78,1 \%$ & $18,8 \%$ & $100 \%$ & \\
\hline
\end{tabular}

Suma de rangos de Wilcoxon $(p>0,05)$ no significativo.

En la tabla 3 se presenta la distribución del grado de aceptabilidad de los adhesivos para prótesis total según los grupos de estudio. Aquí el gel adhesivo a base de Heliocarpus americanus muestra un alto porcentaje en "ni me gusta ni me disgusta" (37,5\%), a diferencia del adhesivo Corega que le "gusta ligeramente" al $50 \%$ de los sujetos de estudio. Siendo $p>0,05$, no se rechaza hipótesis nula; por lo tanto, el grado de aceptabilidad no difiere según las sustancias experimentadas, dado que no hubo diferencias significativas $(p=0,120)$.

\section{DISCUSIÓN}

En la literatura, no hay estudios de investigación del efecto de los adhesivos protésicos a base de la corteza del árbol de Heliocarpus americanus después de la aplicación, especialmente en pacientes.

Las ventajas de los adhesivos, de cómo mejoran la estabilidad protésica y ayudan a mejorar la fonación en los pacientes, se debe a la unión que permite este agente con la mucosa; de modo que los pacientes pueden pronunciar con mucha más energía sin que la prótesis experimente movilidad. Basándonos en los resultados anteriores se puede considerar que el gel natural a base polisacárico (Heliocarpus americanus) ejerce un efecto similar a su versión comercial en la adhesividad de la prótesis total removible en la mucosa de la cavidad bucal. Por lo tanto, beneficios en el ámbito de la rehabilitación oral en pacientes completamente desdentados mejora la calidad de vida de estos; lo cual contrasta con el estudio realizado por De Oliveira ${ }^{(8)}$, quien encontró un aumento significativo en el rendimiento masticatorio después de usar el Ultra Corega crema (media, 32,6\%) y Ultra Corega en polvo (media, 31,2 \%) en comparación con el grupo control (media, 19,8 \%) $(p<0,001)$. La prueba de estabilidad, valorada mediante el método descrito por Woelfel, muestra que en el grupo experimental (Heliocarpus americanus) se logró el valor "aceptable" en un mayor porcentaje; mientras que el adhesivo Corega obtuvo un valor "aceptable" en un 75 $\%$; por lo tanto, no existen diferencias estadísticamente significativas entre ambos adhesivos $(p=0,221)$. De igual manera, la prueba de retención, valorada mediante la escala tomada de Pawan et al. ${ }^{(16)}$ muestra que en el grupo experimental (Heliocarpus americanus) se logró un valor "bueno", mientras que en el adhesivo Corega se obtuvo valor "excelente"; existiendo, por lo tanto, diferencias estadísticamente significativas entre ambos adhesivos $(p=0,004)$.

Al comprobar que los valores de retención y estabilidad son diferentes en los adhesivos utilizados, otros investigadores como Rubilar et al. ${ }^{(17)}$ demostraron

Tabla 3. Grado de aceptabilidad de los adhesivos para prótesis según grupos de estudio

\begin{tabular}{|c|c|c|c|c|c|c|c|}
\hline \multirow[b]{2}{*}{ Sustancias evaluadas } & \multicolumn{5}{|c|}{ Grado aceptado } & \multirow[b]{2}{*}{ Total } & \multirow[b]{2}{*}{$p$} \\
\hline & $\begin{array}{c}\text { Me disgusta } \\
\text { mucho }\end{array}$ & $\begin{array}{l}\text { Me disgusta } \\
\text { ligeramente }\end{array}$ & $\begin{array}{l}\text { Ni me gusta ni } \\
\text { me disgusta }\end{array}$ & $\begin{array}{l}\text { Me gusta } \\
\text { ligeramente }\end{array}$ & $\begin{array}{c}\text { Me gusta } \\
\text { mucho }\end{array}$ & & \\
\hline \multirow{2}{*}{ Heliocarpus americanus } & 0 & 3 & 6 & 4 & 3 & 16 & \\
\hline & $0 \%$ & $18,8 \%$ & $37,5 \%$ & $25 \%$ & $18,8 \%$ & $100 \%$ & 0,120 \\
\hline \multirow{2}{*}{ Grupo control } & 0 & 0 & 3 & 8 & 5 & 16 & \\
\hline & $0 \%$ & $0 \%$ & $18,8 \%$ & $50 \%$ & $31,3 \%$ & $100 \%$ & \\
\hline \multirow{2}{*}{ Total } & 0 & 3 & 9 & 12 & 8 & 32 & \\
\hline & $0 \%$ & $9,4 \%$ & $28,1 \%$ & $37,5 \%$ & $25 \%$ & $100 \%$ & \\
\hline
\end{tabular}

Suma de rangos de Wilcoxon $(p>0,05)$ no significativo. 
que la estabilidad valorada mediante el método descrito por Woelfel muestra que en ambas técnicas logran valores "excelentes" (4 de 4). Así lo demostró, igualmente, el estudio realizado por Koronis et al. ${ }^{(18)}$, en el cual evaluaron tres adhesivos protésicos en pacientes edéntulos con prótesis total, produciendo más satisfacción en los pacientes que los utilizaron, en cuanto a que mejoraban la habilidad masticatoria; sobre todo en los pacientes con menor reborde alveolar y más deficiencias de sujeción.

Nuestros hallazgos respecto a la estabilidad de la prótesis removible con el uso de adhesivo natural fueron similares en comparación al grupo control, con resultados semejantes a estudios anteriores como el de Wang et al. ${ }^{(19)}$, en donde al medir las fuerzas necesarias para remover las prótesis, con y sin la utilización de adhesivos, observaron que los adhesivos mejoraban la retención de ambas prótesis como si fuesen nuevas. Con relación a la presentación del adhesivo protésico en el estudio, se elaboró en forma de gel y su efecto fue similar. Sin embargo, encontramos investigaciones con otro tipo de presentaciones, como los estudios realizado por Chowdry et al. (20), quienes encontraron una reducción significativa del desprendimiento cuando un adhesivo era usado; igualmente encontraron que la forma en pasta era más retentiva que la presentada en polvos, atribuido a la mayor viscosidad. En nuestro país existen pocos reportes al respecto.

Respecto al grado de aceptabilidad, en el adhesivo del gel Heliocarpus americanus resultó un mayor porcentaje de "ni me gusta ni me disgusta" (37,5\%), en comparación a la crema Corega que presentó el parámetro "me gusta ligeramente" en un $50 \%$. Cabe mencionar que este estudio preliminar sobre el efecto del gel natural a base de Heliocarpus americanus, como adhesivo protésico, tiene algunas limitaciones. En primer lugar, dado que el método usado fue subjetivo, impide que las conclusiones de este estudio sean categóricas; por lo que se sugiere en futuras investigaciones ampliar el análisis del efecto del gel natural usando otros métodos, midiendo la fuerza de mordida incisal, considerando el uso de cremas adhesivas a base del Heliocarpus americanus. En segundo lugar, el análisis preclínico de la crema adhesiva natural requiere de la complementación con estudios de toxicidad aguda y crónica que cuenten con una caracterización fitoquímica más amplia, con ayuda de cromatografía de gases con espectrometría de masas.

Por consiguiente, se recomienda ampliar el análisis del efecto del gel natural usando otros métodos, midiendo la fuerza de mordida incisal, considerando el uso de cremas adhesiva a base del Heliocarpus americanus.

A partir de los resultados generales podemos concluir que el gel de la corteza del Heliocarpus americanus (palo de goma) presentó efecto adhesivo para prótesis totales, muestra propiedades adhesivas comparable con el corega, ambos resultaron ser igualmente efectivos.

\section{Agradecimiento}

A los docentes de la Facultad de Farmacia y Bioquímica de la Universidad Nacional Mayor de San Marcos, quienes elaboraron el gel adhesivo para prótesis dental y a los pacientes que participaron de la encuesta.

\section{REFERENCIAS BIBLIOGRÁFICAS}

1. Lima C, Zardo F, Sgavioli E, Mollo F, Compagni M. La influencia de factores sistémicos en el uso de las prótesis. Rev Cub Estomatol [Internet] 2009; 46(1): 1-10 [Consultado 2018 Abr 23] Disponible en: http://scielo.sld.cu/ pdf/est/v46n1/est06109.pdf

2. Coates AJ. Usage of denture adhesives. J Dent. 2000; 28(2): 137-140.

3. Instituto Nacional de Salud. Situación de la población adulta Mayor. Octubre-Noviembre-Diciembre 2017. Informe técnico n.o 1 - Marzo 2018 [Internet] [Consultado año mes día] Disponible en: https://www.inei.gob. pe/media/MenuRecursivo/boletines/01-informe-tecnico-n01_adulto-oct-nov-dic2017.pdf

4. Felton D, Cooper L, Duqum I, Minsley G, Guckes A, Haug $S$, et al. Pautas basadas en evidencia para el cuidado y mantenimiento de dentaduras postizas completas: una publicación del Colegio Americano de Prostodoncistas . JADA. 2011; 142: 1S-20S.

5. Kumar P, Shajahan P, Mathew J, Koruthu A, Aravind P, Ahammed M. Adhesivos para dentaduras postizas en prostodoncia: una visión general. J Int Oral Health. 2015; 7(1): 93-5.

6. Psillakis J, Wright R, Grbic J, Lamster I. En la práctica, evaluación de un adhesivo para dentaduras postizas utilizando un gnatómetro. J Prosthodont. 2004; 13: 244-250.

7. Pawan K, Ramesh N, Farhan K. Una investigación sobre el efecto de los adhesivos para dentaduras postizas sobre la fuerza de mordida incisal de quienes usan dentaduras postizas completas utilizando transductores de presión: un estudio clínico. J Adv Prosthodont. 2012 mayo; 4(2): 97-102.

8. De Oliveira N, Santana N, Mendoza D, Pelean A, De Pero A, Compagnoni M. Desempeño masticatorio de usuarios de prótesis completas después de usar dos adhesivos: un ensayo clínico aleatorizado cruzado. J Prosthet Dent. 2014; 112(5): 1182-7.

9. Mendoza D, Perin A, Palean A, Santana L, de Oliveira N, de Pero A, et al. Efecto de un adhesivo para dentaduras postizas sobre la satisfacción y los parámetros kinesiográficos de los usuarios completos de prótesis: un ensayo clínico aleatorizado cruzado. Braz Dent J. 2014; 25(5): 391-8.

10. Figueiral M, Fonseca P, Pereira C, Scully C. El efecto de diferentes materiales adhesivos sobre la retención de 
las prótesis totales maxilares. Int J. Prosthodont. 2011; 24(2): 175-7.

11. Missouri Botanical Garden (MBG) [Internet] Saint Louis (MO): MBG; 2009. Disponible en: http://www.tropicos. org/

12. Aroca A, Montilla D. Evaluación de la propagación vegetativa del Balso blanco Heliocarpus americanus H.B.K. en la vereda la Merced, municipio de La Unión departamento de Nariño. Pasto: Universidad de Nariño. Facultad de Ciencias Agrícolas; 2006.

13. Vásquez C, Uribe A, Álvarez J. Propagación por estacas juveniles de Balso Blanco (Heliocarpus americanus L. Sin. H. popayanensis) utilizando propagadores de sub-irrigación. Revista Facultad Nacional de Agronomía Medellín. 2006; 59(2): 3479-3498.

14. Alvarado D, Mozombite M. efecto del tiempo de extracción y del pH en la obtención del hidrocoloide de corteza de Huampo (Heliocarpus americanus L.) [Tesis de pregrado] Huánuco: Universidad Nacional Hermilio Valdizán; 2017.

15. Dantas M, Reis S, Damasceno C, Rolim L, Rolim-Neto P, Carvalho F, et al. Development and Evaluation of Stabi- lity of a Gel Formulation Containing the Monoterpene Borneol. Sci World J. 2016; 20: 10-3.

16. Pawan K, Ramesh N, Farhan K. Una investigación sobre el efecto de los adhesivos para dentaduras postizas sobre la fuerza de mordida incisal de quienes usan dentaduras postizas completas utilizando transductores de presión: un estudio clínico. J Adv Prosthodont. 2012; 4(2): 97-102.

17. Rubilar $F$, Jiménez $F$, Rochefort $C$. Retención y estabilidad de bases protésicas superiores obtenidas a partir de dos técnicas de impresión utilizadas en la rehabilitación de dentaduras totales con prótesis convencional. Rev Dental de Chile. 2009; 100(1): 4-12.

18. Koronis S,PizatosE,Polyzois G, Lagouvardos P.Clinicalevaluation of three denture cushion adhesives by complete denture wearers. Gerodontology.2012;29(2):e161-e169.

19. Wang YB, Chen YY. Adhesive mediated enhancement of oclusal force measurements in patients with existing and new complete dentures: a pilot study. Int J Prosthodont. 2010; 23(2): 155-157.

20. Chowdhry P, Phuketa S, Patil R, Yadav H. A study to evaluate the retentive ability of different denture adhesive materials: An in vitro stud. J Indian Prosthodont Soc. 2010; 10(3): 176-181. 